\title{
The Role of Copy Number Variation in African Americans with Type 2 Diabetes-Associated End Stage Renal Disease
}

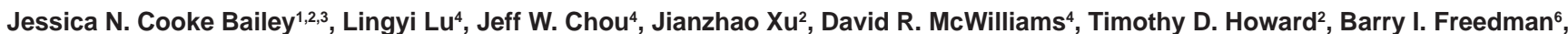
Donald W. Bowden ${ }^{2,3,5,7}$, Carl D. Langefeld ${ }^{4}$ and Nicholette D. Palmer ${ }^{1,3,5}$

${ }^{1}$ Program in Molecular Medicine and Translational Science, Wake Forest School of Medicine, Medical Center Boulevard, Winston-Salem, North Carolina 27157, USA ${ }^{2}$ Center for Genomics and Personalized Medicine Research, Wake Forest School of Medicine, Medical Center Boulevard, Winston-Salem, North Carolina 27157, USA ${ }^{3}$ Center for Diabetes Research, Wake Forest School of Medicine, Medical Center Boulevard, Winston-Salem, North Carolina 27157, USA

${ }^{4}$ Department of Biostatistical Sciences - Division of Public Health Sciences, Wake Forest School of Medicine, Medical Center Boulevard, Winston-Salem, North Carolina 27157, USA

${ }^{5}$ Department of Biochemistry, Wake Forest School of Medicine, Medical Center Boulevard, Winston-Salem, North Carolina 27157, USA

${ }^{6}$ Department of Internal Medicine - Section on Nephrology, Wake Forest School of Medicine, Medical Center Boulevard, Winston-Salem, North Carolina 27157, USA

${ }^{7}$ Department of Internal Medicine - Section on Endocrinology, Wake Forest School of Medicine, Medical Center Boulevard, Winston-Salem, North Carolina 27157, USA

\begin{abstract}
This study investigated the association of copy number variants (CNVs) in type 2 diabetes (T2D) and T2Dassociated end-stage renal disease (ESRD) in African Americans. Using the Affymetrix 6.0 array, $>900,000 \mathrm{CNV}$ probes spanning the genome were interrogated in 965 African Americans with T2D-ESRD and 1029 non-diabetic African American controls. Previously identified and novel CNVs were separately analyzed and were evaluated for insertion/deletion status and then used as predictors in a logistic regression model to test for association. One common CNV insertion on chromosome 1 was significantly associated with T2D-ESRD $\left(p=6.17 \times 10^{-5}, O R=1.63\right)$ after multiple comparison correction. This CNV region encompasses the genes $A M Y 2 A$ and $A M Y 2 B$, which encode amylase isoenzymes produced by the pancreas. Additional common and novel CNVs approaching significance with disease were also detected. These exploratory results require further replication but suggest the involvement of the AMY2A/AMY2B CNV in T2D and/or T2D-ESRD, and indicate that CNVs may contribute to susceptibility for these diseases.
\end{abstract}

Keywords: Copy number variation; African Americans; Diabetic nephropathy; End-stage renal disease; Genome-wide association study; Type 2 diabetes

\section{Introduction}

Diabetes is a complex and heterogeneous disease with a staggering global impact - most recent estimates indicate 346 million people worldwide suffer from this disease [1]. Type 2 diabetes (T2D) is the most common form of diabetes, accounting for more than $90 \%$ of cases, and occurs when peripheral tissue insulin resistance accompanies insufficient $\beta$-cell insulin production. While more than $80 \%$ of diabetes deaths occur in low- and middle-income countries [1], there remains an enormous diabetes burden in the United States as it is the seventh leading cause of death [2]. One of the most detrimental and expensive comorbidities of diabetes is kidney disease (nephropathy), which develops in $\sim 40 \%$ of patients [3] and accounts for $10-20 \%$ of all-cause mortality [1]. The final stage of diabetes-associated nephropathy is end-stage renal disease (ESRD), a complication which occurs more frequently in African Americans than other American populations [4]. T2D and T2D-associated ESRD (T2D-ESRD) are likely influenced by complex interactions between environmental surroundings and lifestyle choices, highlighted by the significant increase in T2D over the last several decades [5]. In addition to environmental factors, numerous genetic loci have been identified as potential mediators of T2D and T2D-ESRD [6-8]. Though these have aided the understanding of the pathogenesis of these diseases, the combined impact of identified genetic contributors is low relative to the heritability of these diseases. While common genetic variants, specifically single nucleotide polymorphisms (SNPs), have been identified and explored in detail as possible mediators, copy number variation $(\mathrm{CNV})$ is a relatively novel source of genetic variation that has only recently been reported as having a potential role in T2D in European-derived and Asian populations [9-11]. Additionally, prior studies of CNVs associated with T2D have not included African Americans [9-11].

Important for this study, recent reports have identified CNVs associated with related metabolic traits and disorders including BMI [12] and obesity [13-15]. CNVs associated with kidney disease (specifically glomerulonephritis) have also been reported [16]. Complex diseases with disparate incidence rates in different race groups are particularly interesting areas to focus attention, as gene dosage may impact disease manifestation [17]. Based on these observations we performed a CNV analysis of T2D and T2D-ESRD in an African American sample.

\section{Materials and Methods}

\section{Subjects}

A total of 965 African Americans with T2D-ESRD and 1029 nondiabetic African American controls were evaluated. Ascertainment criteria and recruitment methods have been described $[18,19]$. Recruitment and sample collection procedures were approved by the Institutional Review Board at Wake Forest School of Medicine and written informed consent was obtained from all participants.

*Corresponding author: Nicholette D. Palmer, Department of Biochemistry, Wake Forest School of Medicine, Medical Center Boulevard,Winston-Salem, NC 27157, USA, Tel: 336-713-7534; Fax: 336-713-7566; E-mail: nallred@wakehealth.edu

Received June 18, 2013; Accepted July 27, 2013; Published July 31, 2013

Citation: Cooke Bailey JN, Lu L, Chou JW, Xu J, McWilliams DR, et al. (2013) The Role of Copy Number Variation in African Americans with Type 2 DiabetesAssociated End Stage Renal Disease. J Mol Genet Med 7: 61. doi:10.4172/17470862.1000061

Copyright: (c) 2013 Cooke Bailey JN, et al. This is an open-access article distributed under the terms of the Creative Commons Attribution License, which permits unrestricted use, distribution, and reproduction in any medium, provided the original author and source are credited 
Case and control subjects were unrelated, self-described African Americans born in North Carolina, South Carolina, Georgia, Virginia or Tennessee. Subjects with T2D-ESRD were recruited from dialysis facilities (case subjects). T2D was diagnosed as developing diabetes at $\geq 25$ years of age without prior diabetic ketoacidosis. Additionally, T2D-ESRD cases met at least one of the following criteria for inclusion: a) T2D diagnosed $\geq 5$ years before initiating renal replacement therapy, b) background or greater diabetic retinopathy and/or c) $\geq 100 \mathrm{mg} / \mathrm{dl}$ proteinuria on urinalysis without other causes of nephropathy. Control subjects without a current diagnosis of diabetes or renal disease were recruited from the community and internal medicine clinics. DNA extraction was performed using the PureGene system (Gentra systems; Minneapolis, MN).

\section{Genotyping and quality control}

Genotyping was performed at the Center for Inherited Disease Research (CIDR) using $1 \mu \mathrm{g}$ of genomic DNA (diluted in $1 \mathrm{X}$ TE buffer and at 50ng/ $\mu \mathrm{l}$ ) on the Affymetrix Genome-wide Human SNP array 6.0 (Affy6.0). Genotyping and quality control have been previously described in depth $[18,19]$.

\section{Analysis}

Principal components analysis (PCA) was performed utilizing all high-quality SNP data from the Affy 6.0; regions of high linkage disequilibrium and inversions were excluded [18,19]. Direct comparison of the PCA with FRAPPE [20] analysis of 70 ancestry informative markers (AIMs) resulted in a high correlation between PC1 and the AIMs $\left(\mathrm{r}^{2}=0.87\right)$. The mean $( \pm \mathrm{SD})$ African ancestry proportions in 965 T2D-ESRD cases and 1,029 controls were $0.80 \pm 0.11$ and $0.78 \pm$ 0.11 , respectively, as estimated by FRAPPE analysis [20]. PCA was also computed on intensity data to detect batch effects; intensity data were normalized using the software Partek [21] prior to CNV calling.

$\mathrm{CNV}$ data was analyzed using Affymetrix Genotyping Console 4.0 software with version 30 library and annotation files. This software implements the Birdsuite program tools to identify common, novel, and rare CNV [22] . Copy number (i.e., insertion or deletion) was estimated at all individual probes (novel CNV) and an a priori set of CNVs previously identified and described in McCarroll et al. [23] and implemented in Birdsuite [22]. Following software recommendations, common CNVs were called using the CANARY algorithm and novel CNVs were called using the hidden Markov models implemented in Birdseye [22]. For each method, probes or regions were categorized by copy number $(\mathrm{CN})$ status with insertions defined as $\mathrm{CN}>2$ and deletions defined as $\mathrm{CN}<2$; probes with $\mathrm{CN}=0$ were considered homozygous deletions. Insertions and deletions present in $<5$ individuals were discarded from further analyses. Following these criteria, for each of the 1,130 previously described CNV regions, 328 insertions and 941 deletions were deemed informative. For individual probes, 113,191 and 134,300 insertions and deletions, respectively, were deemed informative. CN status was coded as a binary variable indicating presence or absence of an insertion/deletion in each sample and used as the predictor in logistic regression models with covariates age, gender, and principal component 1 (PC1) (adjustment for admixture) to test for association with disease. Statistical significance, determined separately for insertions and deletions, was defined by Bonferroni correction. Genomic control inflation factors, based on a single probe when the detected $\mathrm{CNV}$ was estimated to extend over a region in which $\geq 1$ probe identified the same CNV, and P-P plots were evaluated for evidence of systematic bias.

\section{Results}

\section{Clinical characteristics of the study samples}

Table 1 summarizes the clinical characteristics of the 1,994 study samples. Age at enrollment for T2D-ESRD cases was older than the non-diabetic controls; however, age at enrollment for controls was older than age at T2D diagnosis in the cases. The T2D-ESRD cases included a higher proportion of females; additionally, on average, all groups were overweight or obese at enrollment.

\section{Analysis}

In the analysis of previously identified CNVs, 14,570 probes corresponding to 1,130 known $\mathrm{CNV}$ regions [23], including 328 insertions and 941 deletions, met QC parameters and were informative. Bonferroni-corrected thresholds of significance were $\mathrm{P} \leq 1.52 \times 10^{-4}$ and $\mathrm{P} \leq 5.31 \times 10^{-5}$ for previously identified insertions and deletions, respectively. Inflation factors (the mean of the $\mathrm{X}^{2}$ statistic) were computed based on informative regions; these were 1.13 and 0.98 for insertions and deletions, respectively. P-P plots for these suggest there was little evidence of a systematic bias (Figure 1A-B).

One previously described CNV met a strict Bonferroni threshold of $\mathrm{P} \leq 1.52 \times 10^{-4}$; this was an insertion on chromosome 1 spanning $50.15 \mathrm{~kb} ; \mathrm{p}=6.17 \times 10^{-5}$, odd ratio $(\mathrm{OR})=1.63$ (95\% CI $\left.1.28-2.08\right)$. The insertion was common in cases $(0.425)$ and controls $(0.377)$. This region encompasses $A M Y 2 A$ and $A M Y 2 B$, pancreatic amylase precursor genes which encode pancreatic amylase isoenzymes. Table 2 includes details of additional top scoring CNVs from this analysis.

A by-probe analysis was performed to detect novel CNVs; 227, 287 individual probes passed QC metrics and were informative. These included 113,191 insertions and 134,300 deletions, corresponding to Bonferroni-corrected significance thresholds of $\mathrm{P} \leq 4.42 \times 10^{-7}$ and $\mathrm{P} \leq 3.7 \times 10^{-7}$ for insertions and deletions, respectively. Inflation factor calculations were 0.77 and 0.88 for insertions and deletions, respectively. P-P plots are shown in Figure 1C-D. No result from this analysis met strict Bonferroni-corrected significance thresholds; top results are shown in Table 2. The most significant insertion spanned $149.27 \mathrm{~kb}$ on chromosome 17; the best $\mathrm{p}$-value for all probes in this region was $5.91 \times 10^{-4}$, frequencies were 0.091 and 0.147 in cases and controls, respectively, and OR=0.54 (95\% CI 0.38-0.77). Genes in this region include KIAA1267, LOC644246, LRRC37A (leucine rich repeat containing 37A) and $A R L 17 B$ (ADP-ribosylation factor-like 17B). The most significant deletion spanned $247.16 \mathrm{~kb}$ on chromosome 14; the

\begin{tabular}{|l|c|c|c|}
\hline & T2D-ESRD & $\begin{array}{c}\text { Non-diabetic } \\
\text { Controls }\end{array}$ & P-Value \\
\hline$n$ & 965 & 1029 & \\
\hline Female (\%) & 61.2 & 57.3 & 0.076 \\
\hline Age at Enrollment (years) & $61.6 \pm 10.5$ & $49.0 \pm 11.9$ & $<0.0001$ \\
\hline Age at T2D diagnosis (years) & $41.6 \pm 12.4$ & - & $<0.0001^{*}$ \\
\hline Age at ESRD diagnosis (years) & $58.0 \pm 10.9$ & - & \\
\hline T2D to ESRD duration (years) & $16.2 \pm 10.9$ & - & \\
\hline BMI (kg/m²) & $29.7 \pm 7.0$ & $30.0 \pm 7.0$ & 0.30 \\
\hline
\end{tabular}

${ }^{*}$ Comparison of Age at T2D diagnosis in T2D-ESRD cases to Age at Enrollment in Non-diabetic Controls.

Table 1: Clinical characteristics of study samples (values are presented as trait mean \pm standard deviation). 
Citation: Cooke Bailey JN, Lu L, Chou JW, Xu J, McWilliams DR, et al. (2013) The Role of Copy Number Variation in African Americans with Type 2 Diabetes-Associated End Stage Renal Disease. J Mol Genet Med 7: 61. doi:10.4172/1747-0862.1000061

A

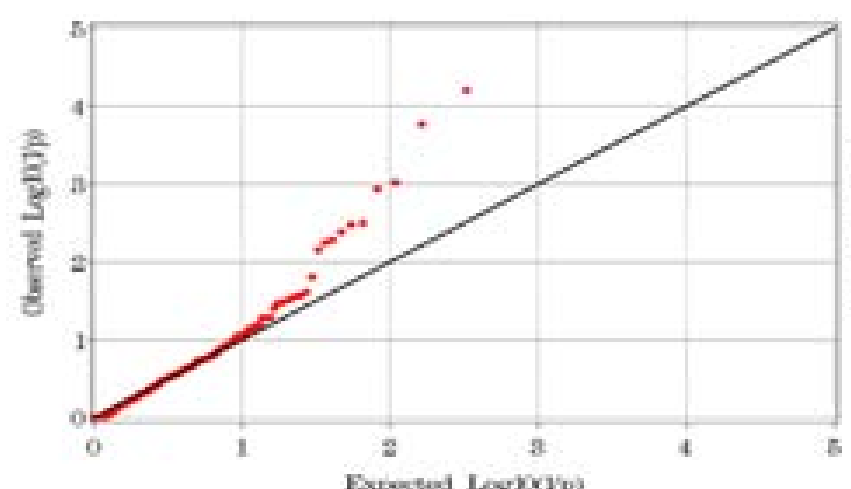

C

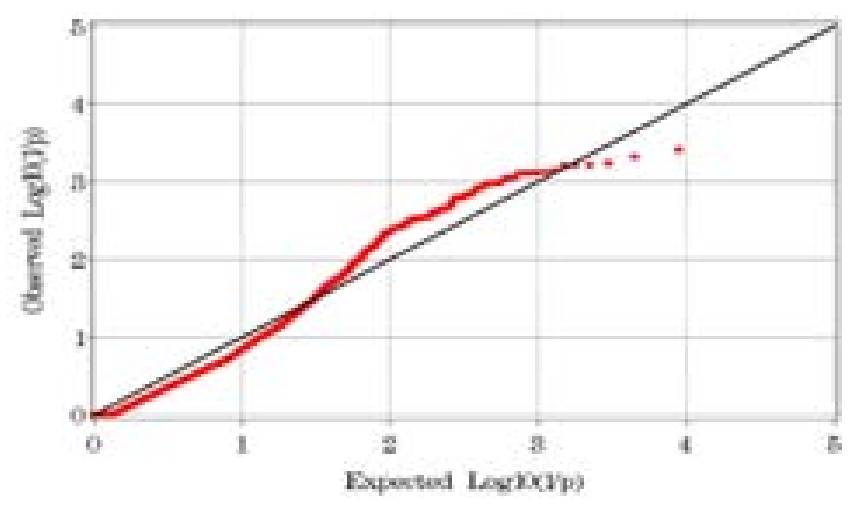

B

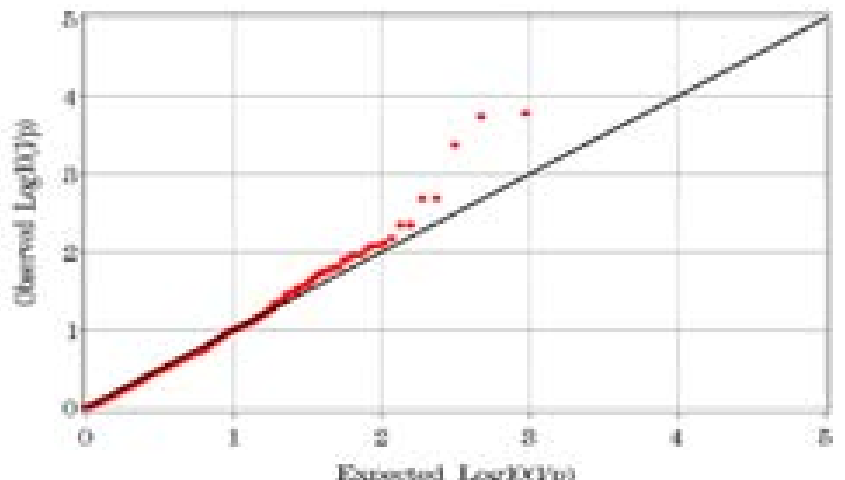

D

Deletion

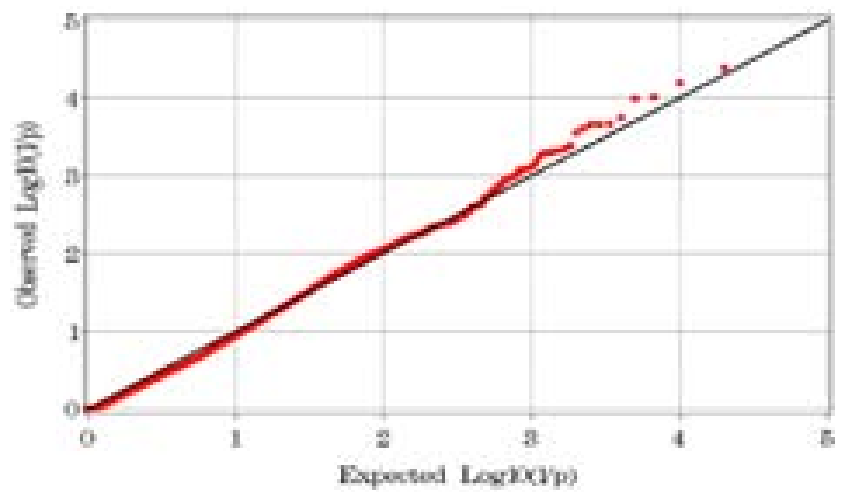

Figure 1: P-P plots for insertion $(A, C)$ and deletion $(B, D)$ models of previously identified $C N V s(A, B)$ and individual $C N V$ probes $(C, D)$.

\begin{tabular}{|c|c|c|c|c|c|c|}
\hline & \multirow{2}{*}{$\begin{array}{c}\text { hg18 Position }{ }^{\infty} \\
\text { chr:start-end }\end{array}$} & \multicolumn{2}{|c|}{ Association } & \multicolumn{2}{|c|}{ Frequency } & \multirow{2}{*}{ Nearest Gene(s) } \\
\hline & & P-value & OR $(95 \% \mathrm{Cl})$ & Cases & Ctrls & \\
\hline \multirow{6}{*}{$\begin{array}{l}\text { Known } \\
\text { Insertions }^{*}\end{array}$} & chr1:103910761-103960907 & $6.17 \times 10^{-5}$ & $1.62(1.28,2.08)$ & 0.425 & 0.377 & RNPC3, AMY2B, LOC648740, AMY2A, AMY1A, AMY1C, AMY1B \\
\hline & chr12:36340414-36394320 & $1.68 \times 10^{-4}$ & $0.59(0.45,0.78)$ & 0.172 & 0.242 & None within $500 \mathrm{~kb}$ \\
\hline & chr17:41521619-41719991 & $9.46 \times 10^{-4}$ & $0.55(0.39,0.78)$ & 0.092 & 0.145 & KIAA1267, LOC644246, LRRC37A, ARL17B \\
\hline & chr13:75007535-75015769 & $1.14 \times 10^{-3}$ & $0.56(0.40,0.80)$ & 0.089 & 0.116 & COMMD6, UCHL3 \\
\hline & chr15:28377089-28536721 & $3.12 \times 10^{-3}$ & $0.44(0.26,0.76)$ & 0.897 & 0.892 & CHRFAM7A \\
\hline & chr8:140673518-140675508 & $3.29 \times 10^{-3}$ & $2.41(1.34,4.34)$ & 0.052 & 0.023 & KCNK9 \\
\hline \multirow{5}{*}{$\begin{array}{l}\text { Known } \\
\text { Deletions }\end{array}$} & chr5:180311316-180350709 & $1.64 \times 10^{-4}$ & $1.55(1.24,1.95)$ & 0.418 & 0.353 & BTNL8, BTNL3, BTNL9 \\
\hline & chr1:187293847-187297986 & $1.78 \times 10^{-4}$ & $3.44(1.81,6.67)$ & 0.057 & 0.041 & None within $500 \mathrm{~kb}$ \\
\hline & chr9:11957036-11965492 & $4.20 \times 10^{-4}$ & $0.35(0.19,0.63)$ & 0.817 & 0.801 & None within $500 \mathrm{~kb}$ \\
\hline & chr12:11113633-11132799 & $2.03 \times 10^{-3}$ & $0.7(0.56,0.88)$ & 0.534 & 0.595 & PRH1, TAS2R19, TAS2R31, TAS2R46, TAS2R43, TAS2R30 \\
\hline & chr18:38133-68539 & $2.05 \times 10^{-3}$ & $3.57(1.59,8.02)$ & 0.04 & 0.021 & ROCK1P1 \\
\hline \multirow{5}{*}{$\begin{array}{l}\text { Novel } \\
\text { Insertions }\end{array}$} & chr17:41700658-41700683 & $5.91 \times 10^{-4}$ & $0.54(0.38,0.77)$ & 0.091 & 0.147 & KIAA1267, LOC644246, LRRC37A, ARL17B \\
\hline & chr7:142159095-142159120 & $3.81 \times 10^{-4}$ & $3.11(1.66,5.83)$ & 0.062 & 0.017 & PRSS1, TRY6, PRSS2 \\
\hline & chr8:12457161-12457186 & $5.71 \times 10^{-4}$ & $0.49(0.32,0.73)$ & 0.058 & 0.093 & FAM66A, DEFB109P1, FAM90A25P, FAM86B2, LONRF1, MIR3926 \\
\hline & chr12:34438874-34438899 & $2.14 \times 10^{-3}$ & $3.27(1.54,6.98)$ & 0.041 & 0.019 & ALG10 \\
\hline & chr10:135199054-135199079 & $2.35 \times 10^{-3}$ & $0.49(0.31,0.77)$ & 0.051 & 0.072 & CYP2E1, SYCE1, SPRNP1 \\
\hline
\end{tabular}


Citation: Cooke Bailey JN, Lu L, Chou JW, Xu J, McWilliams DR, et al. (2013) The Role of Copy Number Variation in African Americans with Type 2 Diabetes-Associated End Stage Renal Disease. J Mol Genet Med 7: 61. doi:10.4172/1747-0862.1000061

Page 4 of 4

\begin{tabular}{|l|l|l|l|l|l|l|}
\hline & chr14:21965715-21965740 & $4.03 \times 10^{-5}$ & $0.07(0.02,0.24)$ & 0.003 & 0.026 & DAD1, ABHD4 \\
\cline { 2 - 7 } & chr1:110027998-110028023 & $1.77 \times 10^{-4}$ & $2.04(1.41,2.96)$ & 0.123 & 0.078 & GSTM4, GSTM2, GSTM1, GSTM5 \\
\cline { 2 - 6 } $\begin{array}{l}\text { Novel } \\
\text { Deletions }\end{array}$ & chr7:142167293-142167318 & $2.15 \times 10^{-4}$ & $0.6(0.46,0.79)$ & 0.169 & 0.259 & PRSS1, TRY6, PRSS2 \\
\cline { 2 - 7 } & chr2:71200196-71200221 & $8.11 \times 10^{-4}$ & $0.34(0.18,0.64)$ & 0.022 & 0.048 & NAGK, MCEE, MPHOSPH10 \\
\cline { 2 - 6 } & chr3:145765721-145765746 & $1.49 \times 10^{-3}$ & $0.17(0.06,0.50)$ & 0.008 & 0.013 & None within $500 \mathrm{~kb}$ \\
\hline
\end{tabular}

${ }^{\infty}$ McCarroll et al. 2008. *Insertions analyzed=328, yielding a Bonferroni-adjusted threshold of $P \leq 1.52 \times 10^{-4}$; deletions analyzed=941, yielding a Bonferroni-adjusted threshold of $P \leq 5.31 \times 10^{-5}$. *Insertion probes analyzed $=113191$, yielding a Bonferroni-adjusted threshold of $P \leq 4.42 \times 10^{-7}$; deletion probes analyzed $=134300$, yielding a Bonferroni-adjusted threshold of $\mathrm{P} \leq 3.7 \times 10^{-7}$. Association results reported are for the best probe in the region encompassed by the probes which span the reported physical position.

Table 2: Top CNV association information.

best $\mathrm{p}$-value for all probes in this region was $4.03 \times 10^{-5}$, frequencies were 0.003 and 0.026 in cases and controls, respectively, and $\mathrm{OR}=0.07$ (95\% CI 0.02-0.24). Genes nearest this region include DAD1 (defender against cell death 1) and ABHD4 (abhydrolase domain containing 4).

Further scrutiny of the probe-level data revealed four regions encompassed by three or more probes spanning $>1 \mathrm{~kb}$ and where overlap of insertions and deletions occurred (Figure 2). Results trended towards association with disease susceptibility/protection. A rare overlapping region on chromosome 4 (Figure 2A) spanning 15.87 $\mathrm{kb}$ had an insertion $\mathrm{p}$-value of $2.25 \times 10^{-2}, \mathrm{OR}=0.74$ and frequency $=0.16$ in cases and 0.19 in controls; the deletion p-value was $1.84 \times 10^{-3}$, $\mathrm{OR}=11.25$, frequency of 0.04 in cases and 0.02 in controls. The gene nearest this CNV was DEFB131, defensin beta 131, which belongs to the beta-defensin family and is highly expressed in the testis and moderately expressed in the prostate and small intestine [24]. Another overlapping region on chromosome 7 (Figure $2 \mathrm{~B}$ ) spanning $13.28 \mathrm{~kb}$ had an insertion p-value of $3.81 \times 10^{-4}, \mathrm{OR}=3.11$, case frequency $=0.06$ and control frequency $=0.03$. This $\mathrm{CNV}$ encompasses $T R Y 6$, which is thought to be a transcribed pseudogene that encodes a protein similar to trypsinogen [24]. Another overlapping region on chromosome 7 (Figure 2C) spaning $124.62 \mathrm{~kb}$ had an insertion p-value of $3.4510^{-3}$, $\mathrm{OR}=0.54$, case frequency $=0.07$ and control frequency $=0.10$; the deletion $\mathrm{p}$-value was $6.92 \times 10^{-4}, \mathrm{OR}=2.15$, case frequency $=0.12$ and control frequency $=0.09$. This $\mathrm{CNV}$ encompasses several genes, including five olfactory receptors. The fourth and more common overlapping region of interest occurred on chromosome 8 (Figure 2D); this region spans $1.70 \mathrm{~kb}$ and had an insertion $\mathrm{p}$-value of $2.58 \times 10^{-2}, \mathrm{OR}=0.60$, case frequency $=0.12$, and control frequency $=0.13$; the deletion $\mathrm{p}$-value was $1.56 \times 10^{-2}, \mathrm{OR}=1.48$, case frequency $=0.16$, control frequency $=0.12$.

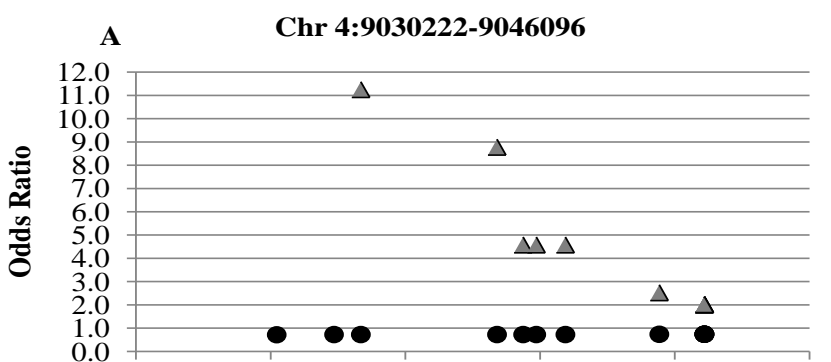

902500090300009035000904000090450009050000

Position along chromosome 4

C Chr 7:143540156-143664772

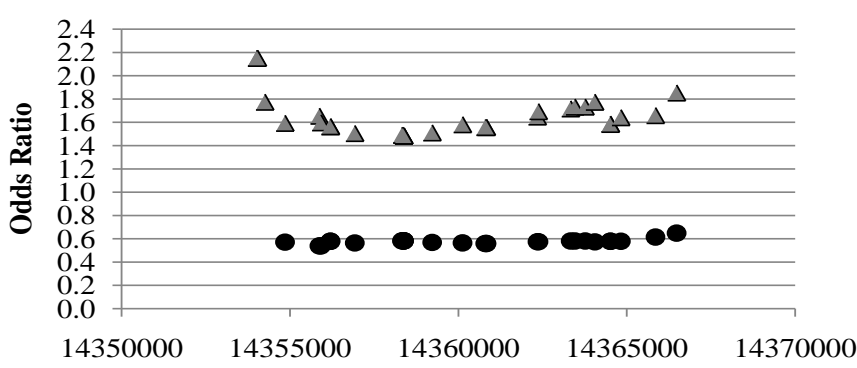

Position along chromosome 7

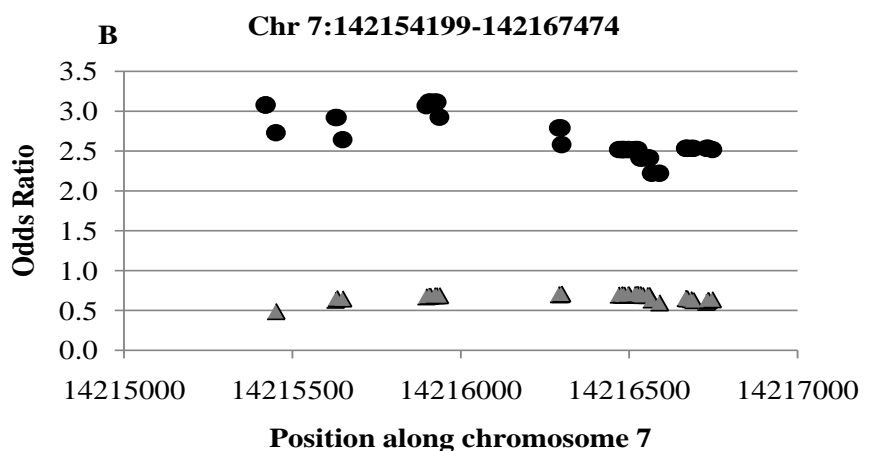

D

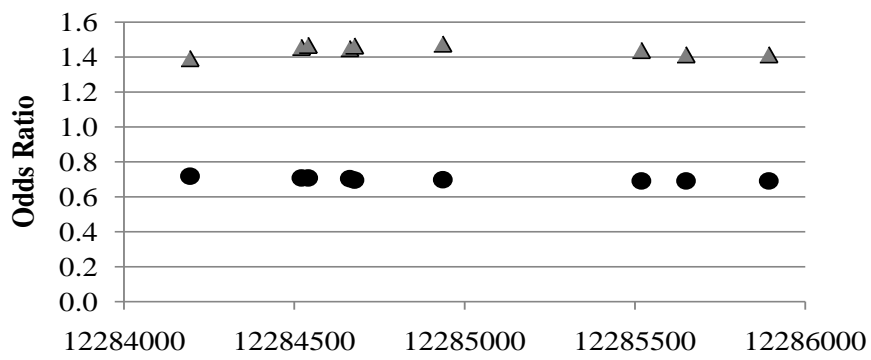

Position along chromosome 8

\section{Insertions \\ $\Delta$ Deletions}

Figure 2: Plots of regions of overlapping CNVs (insertions and deletions) identified in by-probe CNV analysis. The OR of each probe is represented with annotation of insertion or deletion status. 
The gene nearest this $\mathrm{CNV}$ is predicted gene FAM66A, family with sequence similarity 66 , member A.

\section{Discussion}

We performed a GWAS of common and novel CNV data obtained from the Affy 6.0 GWAS array in African Americans with T2D-ESRD. This array was designed to interrogate samples using 946K copy number probes, including 800,000 probes for uniform coverage across the genome and 140,000 additional probes for detection in regions of previously reported CNVs [23], and likely has better coverage of CNVs than other arrays utilized for CNV analyses in T2D. This allowed for two comprehensive analyses. First we examined previously identified common CNVs, a method that is useful for determining if known CNVs are associated with disease and if they exist in the population of interest. Secondly, we performed an unbiased genome-wide analysis based on individual probes, which theoretically enables the detection of undocumented CNVs. Both methods are particularly informative, as African Americans have not been included in prior studies that investigated the potential role of CNVs in T2D or T2D-ESRD.

A single $\mathrm{CNV}$ in a $50 \mathrm{~kb}$ region encompassing the $A M Y 2 A$ and $A M Y 2 B$ genes on chromosome 1 met Bonferroni-corrected thresholds of significance. $A M Y 2 A$ and $A M Y 2 B$ are pancreatic alpha amylase genes encoding secreted proteins that hydrolyze 1, 4-alpha-glucoside bonds in oligosaccharides and polysaccharides, catalyzing the initial step in digestion of dietary starch and glycogen. Both genes are expressed in the pancreas and $A M Y 2 B$ is also expressed in the liver. These characteristics suggest that the region is more likely associated with T2D risk. The region encompassed by this CNV contains more than 20 SNPs including several synonymous and nonsynonymous polymorphisms, intronic enhancers, and splice sites. Additionally, this CNV is within $50 \mathrm{~kb}$ of a previously identified area of common $\mathrm{CNV}$ in the salivary amylase gene AMY1, [25], although these have not been reported in disease studies. Our results suggest a potential role for disruption or duplication of these genes in the development of T2D and/or T2D-ESRD.

We additionally detected known and novel CNVs that occur at appreciable frequencies and approach statistically significant association with T2D/T2D-ESRD in this sample. These included four overlapping regions covering $>1 \mathrm{~kb}$ where insertions and deletions trended towards association in opposite directions of effect. These regions likely represent true copy number events with a potential role in T2D and/or T2D-ESRD and warrant further investigation and replication.

A limitation of this report is that we compared cases with T2D-ESRD to non-nephropathy, non-diabetic controls. Therefore, association may be due to association with T2D or nephropathy. Unfortunately, appreciable numbers of African Americans with longstanding T2D lacking nephropathy or microalbuminuria are difficult to recruit due to the high prevalence of nephropathy in this population. Such individuals would comprise an ideal comparison group, useful for clarifying whether associations resulted from the presence of T2D or nephropathy. Additional limitations include the lack of replication in this study, which is required for the incorporation of this information into the current working model of the genetics of T2D/T2D-ESRD.

A previous study identified 1,362 CNVs in African Americans using the Affymetrix $500 \mathrm{~K}$ array and detected two regions with significant frequency differences between African Americans and European Americans [26]. Using the Affy 6.0 array, we also detected that CNV events were common in this population. Another GWAS employing the Affy6.0 did not detect genome-wide significance between CNVs and anthropometric traits in African Americans [27]. Furthermore, a study that included African American children detected association between CNVs and childhood obesity [13]. While these represent efforts towards incorporating African Americans in studies of alternative genetic contributors to widely studied traits and diseases, there are currently no published reports investigating the role of CNVs in T2D in African Americans.

\section{Conclusions}

In summary, we observed a previously identified insertion on chromosome 1 associated with disease in a large African American sample with T2D-ESRD, which suggests a potential role for disruption or duplication of the encompassed genes in T2D and/or T2D-ESRD. Additionally, we detected that other common and novel CNVs were present at an appreciable frequency and may contribute to risk of T2D and/or T2D-ESRD in this population. These data are exploratory as CNVs represent a novel yet poorly understood form of genetic variation; validation is crucial for elucidation of additional mechanisms contributing to the etiology of these diseases.

\section{Acknowledgements}

Genotyping services were provided by the Center for Inherited Disease Research (CIDR), which is fully funded through a federal contract from the National Institutes of Health $(\mathrm{NIH})$ to The Johns Hopkins University, contract number HHSC268200782096C. This work was supported by NIH grants K99 DK081350 (NDP), R01 DK066358 (DWB), R01 DK053591 (DWB), R01 HL56266 (BIF), R01 DK 070941 (BIF), R01 DK 084149 (BIF) and in part by a grant from the Genera Clinical Research Center of the Wake Forest School of Medicine, M01 RR07122.

\section{References}

1. WHO (2011) WHO Diabetes Fact sheet $\mathrm{N}^{\circ} 312$

2. CDC (2011) National Diabetes Fact Sheet: national estimates and general information on diabetes and pre diabetes in the United States. Department of Health and Human Services, Centers for Disease Control and Prevention, USA

3. de Boer IH, Rue TC, Hall YN, Heagerty PJ, Weiss NS, et al. (2011) Tempora trends in the prevalence of diabetic kidney disease in the United States. JAMA 305: 2532-2539.

4. USRDS (2009) USRDS 2009 Annual Data Report.

5. Das SK, Elbein SC (2006) The Genetic Basis of Type 2 Diabetes. Cell science 2: 100-131.

6. Prokopenko I, McCarthy MI, Lindgren CM (2008) Type 2 diabetes: new genes, new understanding. Trends Genet 24: 613-621.

7. Prokopenko I, Langenberg C, Florez JC, Saxena R, Soranzo N, et al. (2009) Variants in MTNR1B influence fasting glucose levels. Nat Genet 41: 77-81.

8. Dupuis J, Langenberg C, Prokopenko I, Saxena R, Soranzo N, et al. (2010) New genetic loci implicated in fasting glucose homeostasis and their impact on type 2 diabetes risk. Nat Genet 42: 105-116.

9. Craddock N, Hurles ME, Cardin N, Pearson RD, Plagnol V, et al. (2010) Genome-wide association study of CNVs in 16,000 cases of eight common diseases and 3,000 shared controls. Nature 464: 713-720.

10. Jeon JP, Shim SM, Nam HY, Ryu GM, Hong EJ, et al. (2010) Copy number variation at leptin receptor gene locus associated with metabolic traits and the risk of type 2 diabetes mellitus. BMC Genomics 11: 426.

11. Bae JS, Cheong HS, Kim JH, Park BL, Kim JH, et al. (2011) The genetic effect of copy number variations on the risk of type 2 diabetes in a korean population. PLoS One 6: e19091.

12. Sha BY, Yang TL, Zhao LJ, Chen XD, Guo Y, et al. (2009) Genome-wide association study suggested copy number variation may be associated with body mass index in the Chinese population. J Hum Genet 54: 199-202.

13. Glessner JT, Bradfield JP, Wang K, Takahashi N, Zhang H, et al. (2010) A 
Citation: Cooke Bailey JN, Lu L, Chou JW, Xu J, McWilliams DR, et al. (2013) The Role of Copy Number Variation in African Americans with Type 2 Diabetes-Associated End Stage Renal Disease. J Mol Genet Med 7: 61. doi:10.4172/1747-0862.1000061

Page 6 of 4

genome-wide study reveals copy number variants exclusive to childhood obesity cases. Am J Hum Genet 87: 661-666.

14. Walters RG, Jacquemont S, Valsesia A, de Smith AJ, Martinet D, et al. (2010) A new highly penetrant form of obesity due to deletions on chromosome 16p11.2. Nature 463: 671-675.

15. Wang K, Li WD, Glessner JT, Grant SF, Hakonarson H, et al. (2010) Large copy number variations are enriched in cases with moderate to extreme obesity. Diabetes 59: 2690-2694.

16. Aitman TJ, Dong R, Vyse TJ, Norsworthy PJ, Johnson MD, et al. (2006) Copy number polymorphism in Fcgr3 predisposes to glomerulonephritis in rats and humans. Nature 439: 851-855.

17. Stranger BE, Forrest MS, Dunning M, Ingle CE, Beazley C, et al. (2007) Relative impact of nucleotide and copy number variation on gene expression phenotypes. Science 315: 848-853.

18. McDonough CW, Palmer ND, Hicks PJ, Roh BH, An SS, et al. (2011) A genome-wide association study for diabetic nephropathy genes in African Americans. Kidney Int 79: 563-572.

19. Palmer ND, McDonough CW, Hicks PJ, Roh BH, Wing MR, et al. (2012) A Genome-wide association search for type 2 diabetes genes in african americans. PLoS One 7: e29202.
20. Tang H, Peng J, Wang P, Risch NJ (2005) Estimation of individual admixture: analytical and study design considerations. Genet Epidemiol 28: 289-301.

21. Partek: Next Generation Sequencing \& Microarray Software.

22. Korn JM, Kuruvilla FG, McCarroll SA, Wysoker A, Nemesh J, et al. (2008) Integrated genotype calling and association analysis of SNPs, common copy number polymorphisms and rare CNVs. Nat Genet 40: 1253-1260.

23. McCarroll SA, Kuruvilla FG, Korn JM, Cawley S, Nemesh J, et al. (2008) Integrated detection and population-genetic analysis of SNPs and copy number variation. Nat Genet 40: 1166-1174.

24. UCSC Genome Bioinformatics.

25. Perry GH, Dominy NJ, Claw KG, Lee AS, Fiegler H, et al. (2007) Diet and the evolution of human amylase gene copy number variation. Nat Genet 39: 1256-1260.

26. McElroy JP, Nelson MR, Caillier SJ, Oksenberg JR (2009) Copy number variation in African Americans. BMC Genet 10: 15

27. Kang SJ, Chiang CW, Palmer CD, Tayo BO, Lettre G, et al. (2010) Genomewide association of anthropometric traits in African- and African-derived populations. Hum Mol Genet 19: 2725-2738. 